\title{
A BRIEF PROOF OF JACOBIAN HYPOTHESIS IMPLIES FLATNESS
}

\author{
MASAYOSHI MIYANISHI, LORENZO ROBBIANO, \\ AND STUART SUI-SHENG WANG
}

(Communicated by Louis J. Ratliff, Jr.)

\begin{abstract}
Let $S$ be a polynomial ring in $n$ variables over a field, and let $R$ be the subring generated by $n$ polynomials. We give a short proof of the fact that if the Jacobian determinant of these $n$ polynomials is 1 , then $S$ is a flat $R$-module.
\end{abstract}

Let $S=k\left[x_{1}, \ldots, x_{n}\right]$ be a polynomial ring over a field $k$ and let $f_{1}, f_{2}$, $\ldots, f_{n} \in k\left[x_{1}, \ldots, x_{n}\right]$ satisfy the Jacobian hypothesis

$$
\operatorname{det}\left[\begin{array}{ccc}
\frac{\partial f_{1}}{\partial x_{1}} & \ldots & \frac{\partial f_{1}}{\partial x_{n}} \\
\vdots & \ddots & \vdots \\
\frac{\partial f_{n}}{\partial x_{1}} & \cdots & \frac{\partial f_{n}}{\partial x_{n}}
\end{array}\right]=1
$$

Then $f_{1}, f_{2}, \ldots, f_{n}$ are algebraically independent over $k$ so that $R=$ $k\left[f_{1}, \ldots, f_{n}\right]$ is also a polynomial ring over $k$. If $k$ has characterisitc 0 , the Jacobian conjecture asserts that $S=R$, or equivalently, $S$ is a projective $R$-module [5, Theorem 46 (iii), pp. 483-484]. The Jacobian hypothesis (*) implies that $S$ is smooth over $R$ [4, Theorem, p. 484] and separable over $R$ [5, Theorem 7, p. 458]; moreover, $S$ is finitely generated, equidimensional over $R, R$ is regular, and $S$ is Cohen-Macaulay, hence by Grothendieck's results whose proofs are not easy [1, IV, $\S 15 ; 2, I V, \S 17], S$ is flat over $R$ [5, Theorem $38,(1),(4)$, p. 477]. In this short note we shall give a brief proof of this fact. Consequences of flatness include that the projective dimension of $S$ over $R$ is at most one and that $S \cap k\left(f_{1}, \ldots, f_{n}\right)=R$ [5, pp. 479-481].

Received by the editors July 24, 1989 and, in revised form, November 7, 1989.

1980 Mathematics Subject Classification (1985 Revision). Primary 13F20, 13C11; Secondary 13H05, 14E40, 16A03.

Key words and phrases. Jacobian conjecture, Jacobian hypothesis, smooth algebra, flat module, regular local ring, regualr system of parameters. 
Theorem. If the Jacobian determinant

$$
\frac{\partial\left(f_{1}, \ldots, f_{n}\right)}{\partial\left(x_{1}, \ldots, x_{n}\right)}=1
$$

then $S=k\left[x_{1}, \ldots, x_{n}\right]$ is flat over $R=k\left[f_{1}, \ldots, f_{n}\right]$.

Proof. In order to show that $S$ is flat over $R$, we may assume that $k$ is algebraically closed. To see this let $\bar{k}$ denote the algebraic closure of $k$, then we have by the theorem that $\bar{k}\left[x_{1}, \ldots, x_{n}\right]$ is flat over $\bar{k}\left[f_{1}, \ldots, f_{n}\right]$ and consequently over $k\left[f_{1}, \ldots, f_{n}\right]$. As $\bar{k}$ is free over $k, \bar{k}\left[x_{1}, \ldots, x_{n}\right]$ is faithfully flat over $k\left[x_{1}, \ldots, x_{n}\right]$ and we have that $k\left[x_{1}, \ldots, x_{n}\right]$ is flat over $k\left[f_{1}, \ldots, f_{n}\right]$.

To show $S$ is flat over $R$ it sufficies to show that for all maximal ideals $\mathfrak{q}$ of $S$, the localization $S_{\mathfrak{q}}$ is flat over $R_{\mathfrak{p}}$ where $\mathfrak{p}=\mathfrak{q} \cap R[3$, Theorem 7.1, p. 46]. A maximal ideal $\mathfrak{q}$ of $S$ looks like $\left(x_{1}-\lambda_{1}, \ldots, x_{n}-\lambda_{n}\right)$ for some $\lambda_{1}, \ldots, \lambda_{n} \in$ $k$, and $\mathfrak{p}=\mathfrak{q} \cap R=\left(f_{1}\left(x_{1}, \ldots, x_{n}\right)-f_{1}\left(\lambda_{1}, \ldots, \lambda_{n}\right), \ldots, f_{n}\left(x_{1}, \ldots, x_{n}\right)-\right.$ $\left.f_{n}\left(\lambda_{1}, \ldots, \lambda_{n}\right)\right)$ is a maximal ideal of $R$. To simplfy the notation, let $A=R_{\mathfrak{p}}$, $\mathfrak{m}=\mathfrak{p} R_{\mathfrak{p}}$ its maximal ideal; similarly, let $B=S_{\mathfrak{q}}, \mathfrak{n}=\mathfrak{q} S_{\mathfrak{q}}$ its maximal ideal.

It is well known that $(B, \mathfrak{n}, k)$ is an $n$-dimensional regular local ring so that its maximal ideal $\mathfrak{n}$ can be generated by $n$ elements, any such $n$ elements are called a regular system of parameters of $B$. Moreover, if $\left(v_{1}, \ldots, v_{n}\right) B=\mathfrak{n}$, then there is an isomorphism of graded rings:

$$
\begin{aligned}
k\left[t_{1}, \ldots, t_{n}\right] & \rightarrow \operatorname{gr}_{\mathfrak{n}}(B) \\
t_{i} & \mapsto v_{i}+\mathfrak{n}^{2}
\end{aligned}
$$

for all $i$. Here $k\left[t_{1}, \ldots, t_{n}\right]$ is a polynomial ring over $k$ and

$$
\operatorname{gr}_{\mathfrak{n}}(B)=\bigoplus_{i=0}^{\infty} \frac{\mathfrak{n}^{i}}{\mathfrak{n}^{i+1}}
$$

is the graded ring of $B$ associated with powers of $\mathfrak{n}$.

Similarly, $(A, \mathfrak{m}, k)$ is an $n$-dimensional regular local ring so that if $\mathfrak{m}=$ $\left(u_{1}, \ldots, u_{n}\right) A$, then there is an isomorphism of graded rings: $k\left[t_{1}, \ldots, t_{n}\right] \rightarrow$ $\operatorname{gr}_{\mathrm{m}}(A)$ given by $t_{i} \mapsto u_{i}+\mathrm{m}^{2}$ for all $i$.

In what follows, let

$$
u_{i}=\frac{f_{i}\left(x_{1}, \ldots, x_{n}\right)-f_{i}\left(\lambda_{1}, \ldots, \lambda_{n}\right)}{1}
$$

which is an element of $A$. Clearly, $\left(u_{1}, \ldots, u_{n}\right) A=\mathfrak{m}$. Next we want to show that the Jacobian hypothesis $(*)$ implies that $\left(u_{1}, \ldots, u_{n}\right) B=\mathfrak{n}$, i.e., a (hence any) regular system of parameters of $A$ is also a regular system of parameters of $B$, i.e.,

$$
\mathfrak{m} B=\mathfrak{n} .
$$


Using Taylor expansion, we have the following matrix equation:

$$
\left[\begin{array}{c}
f_{1}\left(x_{1}, \ldots, x_{n}\right)-f_{1}\left(\lambda_{1}, \ldots, \lambda_{n}\right) \\
\vdots \\
f_{n}\left(x_{1}, \ldots, x_{n}\right)-f_{n}\left(\lambda_{1}, \ldots, \lambda_{n}\right)
\end{array}\right] \equiv\left[\begin{array}{ccc}
\frac{\partial f_{1}}{\partial x_{1}}(\vec{\lambda}) & \ldots & \frac{\partial f_{1}}{\partial x_{n}}(\vec{\lambda}) \\
\vdots & \ddots & \vdots \\
\frac{\partial f_{n}}{\partial x_{1}}(\vec{\lambda}) & \ldots & \frac{\partial f_{n}}{\partial x_{n}}(\vec{\lambda})
\end{array}\right]\left[\begin{array}{c}
x_{1}-\lambda_{1} \\
\vdots \\
x_{n}-\lambda_{n}
\end{array}\right]\left(\bmod q^{2}\right) .
$$

Here $\vec{\lambda}=\left(\lambda_{1}, \ldots, \lambda_{n}\right)$. Passing to the localization $S_{\mathrm{q}}$, we have

$$
\left[\begin{array}{c}
u_{1} \\
\vdots \\
u_{n}
\end{array}\right] \equiv\left[\begin{array}{ccc}
\frac{\partial f_{1}}{\partial x_{1}}(\vec{\lambda}) & \ldots & \frac{\partial f_{1}}{\partial x_{n}}(\vec{\lambda}) \\
\vdots & \ddots & \vdots \\
\frac{\partial f_{n}}{\partial x_{1}}(\vec{\lambda}) & \cdots & \frac{\partial f_{n}}{\partial x_{n}}(\vec{\lambda})
\end{array}\right]\left[\begin{array}{c}
\frac{x_{1}-\lambda_{1}}{1} \\
\vdots \\
\frac{x_{n}-\lambda_{n}}{1}
\end{array}\right] \quad\left(\bmod n^{2}\right)
$$

Since the Jacobian matrix is invertible by $(*)$ the last equation shows that the images of $u_{1}, \ldots, u_{n}$ form a basis of the vector space $n / n^{2}$ over $k$. Hence by a version of NAK (Nakayama's lemma) [3, Theorem 2.3 (i), p. 8], $u_{1}, \ldots, u_{n}$ generate $\mathfrak{n}$. This establishes $(* *)$.

As a result of $(* *)$, we have the following commutative diagram

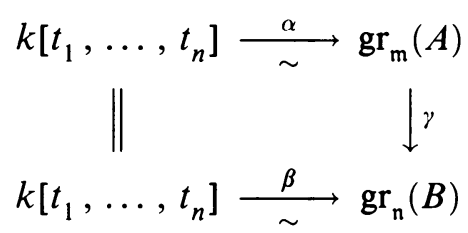

where $\alpha: t_{i} \mapsto u_{i}+\mathfrak{m}^{2}$ and $\beta: t_{i} \mapsto u_{i}+\mathfrak{n}^{2}$ are isomorphisms, and $\gamma$ is induced by the inclusion $A \hookrightarrow B$. Thus $\gamma$ is an isomorphism, and $B$ is flat over $A$ by the local flatness criterion [4, Theorem 22.3, (1), (4'), p. 174].

\section{REFERENCES}

1. A. Grothendieck and J. Dieudonné, Éléments de géométrie algébrique IV, Étude Locale des Schémas et des Morphismes de Schémas (Troisième Partie), Publ. Math., Inst. Hautes Études Sci. 28 (1966).

2. É Éléments de géométrie algébrique IV, Étude Locale des Schémas et des Morphismes de Schémas (Quatrième Partie), Publ. Math., Inst. Hautes Études Sci. 32 (1967).

3. H. Matsumura, Commutative ring theory, Cambridge University Press, Cambridge, 1986. 
4. R. A. Morris and S. S.-S. Wang, A Jacobian criterion for smoothness, J. Algebra 69 (1981), 483-486.

5. S. S.-S. Wang, A Jacobian criterion for separability, J. Algebra 65 (1980), 453-494.

Department of Mathematics, Osaka University, Toyonaka, Osaka 560, Japan

Department of Mathematics, University of Genova, Genova, Italy

Department of Mathematical Sciences, Oakland University, Rochester, Michigan 48309 and Department of Mathematics, Cornell University, Ithaca, New York 14853 